\title{
Sistema de captación de datos del funcionamiento de motores para la toma de decisiones en empresas de producción
}

\author{
Fecha de recepción: 18-12-2020 • Fecha de aceptación: 15-02-2021 • Fecha de publicación: 10-06-2021
}

\author{
Ángel Arturo Flores Lescano \\ Universidad Regional Autónoma de Los Andes, Ecuador \\ angelflow.Ip@gmail.com \\ https://orcid.org/0000-0003-2151-4740
}

\section{RESUMEN}

El presente trabajo tiene como objetivo proponer un sistema para la captación, procesado y almacenamiento de datos en la monitorización del estado de funcionamiento de motores eléctricos para la toma de decisiones en el departamento de manufactura en empresas de producción. Se realiza un análisis de lo existente y se sintetiza verificando la mejor opción para monitorear el funcionamiento de motores. Se utilizaron placas electrónicas con sensores, microcontrolador, comunicación Bluetooth y almacenamiento de datos en una aplicación móvil. Se ha proporcionado a la aplicación la posibilidad de realizar estadística con los datos para anticiparse a fallos que produzcan paros en la producción. El sistema desarrollado (Sistema de Adquisición de Datos de motores, o sus siglas SADAM) en este trabajo tiene la ventaja frente a los sistemas comerciales existentes de ser fácilmente instalado sin parar la producción, no necesitar de ningún sistema previo y ser reconfigurable. Los subsistemas desarrollados se pueden modificar para adaptarse a la monitorización del estado de otros equipos que necesiten medir distintas variables mediante sensores.

PALABRAS CLAVE: captación de datos, funcionamiento de motores eléctricos, toma de decisiones, supervisión. 


\section{ABSTRACT}

The objective of this work is to propose a system for the collection, processing and storage of data in the monitoring of the operating status of electric motors for decision making in the manufacturing department in production companies. An analysis of the existing system is carried out and synthesized to verify the best option to monitor the operation of motors. Electronic boards with sensors, microcontroller, Bluetooth communication and data storage in a mobile application were used. The application has been provided with the possibility of performing statistics with the data to anticipate failures that produce production stoppages. The system developed (Engine Data Acquisition System, or its acronym SADAM) in this work has the advantage over existing commercial systems of being easily installed without stopping production, not needing any previous system and being reconfigurable. The developed subsystems can be modified to adapt to the monitoring of the status of other equipment that needs to measure different variables by means of sensors. 


\section{Introducción}

La captación de datos del funcionamiento de elementos en los procesos de producción es también una parte integral de la industria 4.0 y el Internet de las cosas (IOT), esto se ha hecho una necesidad en las empresas tales como petroquímicas, papeleras, metalúrgicas, centrales de generación, plantas de tratamiento de aguas, incineradoras o la industria farmacéutica, así mejorando la identificación de fallos, tomando decisiones sobre cambio o mantenimiento de dichos elementos partes de la producción, con lo cual realizar mejores trabajos productivos con más eficiencia y mayor eficacia, por ejemplo, Kimberly Clark Costa Rica, en su planta de Belén, se dedica a la elaboración de productos de papel utilizando como base papel de reciclaje. Los procesos de fabricación de papel son complejos y delicados, por esa razón la firma decidió automatizar algunos de los principales, utilizando productos de la línea de Foxboro. En Kimberly Clark se ha llegado con ellos hasta el nivel de uso del SCADA (Supervisory Control And Data Acquisition) Supervisión, Control y Adquisición de Datos. Este sistema es una interfaz que le permite al operador no solo observar distintas variables, sino hacerlas interactuar con el proceso, introduciendo ajustes desde el computador en su cuarto de control (Pérez-López, 2015).

El principal problema que solucionan los sistemas de adquisición de datos y presentación de los mismos al jefe técnico es la inmediatez de la información y la rapidez para detectar inconvenientes que pueden provocar a largo tiempo problemas más serios en cuanto a producción y/o cambios de maquinaria por estado defectuoso. Hoy en día existe mucho interés en utilizar y aplicar técnicas de monitorización de estado (Condition Monitoring) a equipos eléctricos de potencia como transformadores, generadores y motores, utilizando dichas técnicas se consigue reducir los costes de operación y mantenimiento, incrementando la fiabilidad de los equipos (Mariño, Poza, Ubeira, \& Machado, 2004).

El avance tecnológico no puede quedar de lado en ningún tipo de empresa, el desarrollo informático, tanto en el software y hardware, hace que cada vez los sistemas queden obsoletos, $s$ los procesos de adquisición de datos se los realizaba en MS-DOS. En la actualidad, es de imperiosa importancia la renovación tecnológica para que sea una prelación competitiva con el resto de empresas. Tanto es así que, la empresa Centro de Ingeniería Genética y Biotecnología (CIGB), se tomó como referencia los sistemas viejos y mejoraron las limitantes que presentaban estos. Los nuevos sistemas, desde su fecha de concepción, hasta el presente, se han actualizado anualmente en cuanto a versión del software SCADA, versión del sistema operativo y reemplazo de nuevas interfaces, monitoreo de nuevos equipos, inclusión de nuevas variables, entre otros (Martell, Paneque \& Cuesta, 2016).

En empresas de producción se recomienda proceder a la instalación de un sistema móvil que capte datos del funcionamiento de las máquinas y poder tomar decisiones por parte del jefe técnico. En la actualidad, comúnmente las empresas se encuentran experimentando una serie de cambios importantes como: mejoramiento continuo, certificación ISO, implementación de un ERP (Enterprise Resource Planning); los mismos que abren a la compañía nuevas posibilidades de mejorar su eficiencia y competitividad en el mercado, para alcanzar su meta, contar con un sistema de información para monitorear máquinas en el área de producción, lo que es una amplia 
evidencia de la importancia de la tecnología como mecanismo de competitividad empresarial. Las organizaciones que son conscientes y adoptan las Tecnologías de Información y Comunicación (TIC), cuentan con personal especializado, tienen un cierto grado de sistematización y generan planes de contingencia, son más proclives a ser competitivas a escala no solo nacional sino internacional (Ibarra Cisneros et al., 2017).

El control y monitoreo de procesos es fundamental en las industrias, los sistemas SCADA facilitan este trabajo, pero en su mayoría están ligados a un único proveedor de hardware y con software propietario limitando la oferta. Las soluciones actuales funcionan en una PC de escritorio, brindan interface móvil, pero necesitan el sistema de adquisición y control completo ya instalado, con los altos costos y paros largos en la producción en su instalación, provocan la decepción de empresas de mediana producción (Gálvez, 2018).

Cuando se trata de toma de decisiones sobre repuestos e insumos, es de primordial importancia saber que ha ocurrido en cuanto al funcionamiento de cada elemento del área en la cual se trabaja, en esto no sirve documentación estadística externa o tiempo de vida de la máquina estimada por el fabricante, ya que no todas las áreas de producción de todas las empresas son las mismas, y peor aún, el laboratorio de pruebas del fabricante es esencial para registrar todos los datos en un historiador para futuras referencias en una ubicación centralizada para mantener al operador actualizado a través de la interfaz hombre - máquina, y esto brinde verdaderas capacidades de control estadístico remoto en la toma de decisiones. Los sistemas de apoyo a la toma de decisiones son las herramientas que usan los directivos para tomar disposiciones eficaces, basándose en la teoría de la decisión. Por su parte, se puede considerar a las herramientas de captación de datos como tipos especiales de herramientas de apoyo a esta toma, cuando estos datos se procesen y sean información (Marcano \& Talavera, 2007).

LabView es un lenguaje de programación que ha sido completamente adoptado en la industria, la academia y laboratorios de investigación, como el estándar para la adquisición de datos y el software de control de instrumentos. Conjuntamente con el estándar de comunicaciones OPC (OLE for Process Control) de National Instruments, basados en una tecnología Microsoft, lo que permitiría además de la adquisición de datos en la industria el control de esas variables (Salazar \& Arango, 2017).

Arduino es una plataforma electrónica de código abierto basada en hardware y software.

Permite leer entradas, tanto analógicas, como digitales provenientes de sensores, procesar esa información y realizar acciones como encender luces, activar motores, enviar información al Internet, etc. A través de sus pines que también pueden comportarse como salidas (Thompson, 2019).

Hoy día, Arduino está presente en todo tipo de dispositivos tecnológicos, impresoras 3D, dispositivos médicos, aplicaciones domóticas, robots, drones y toda clase de proyectos electrónicos. Basándose en el alto costo de la instalación de un SCADA, Arduino se trata de placas económicas, además, tanto su software y hardware se distribuyen de forma libre, lo que permite abaratar costos y competir con las grandes empresas a nivel mundial (Millahual, 2017). 
Para la programación del Arduino se utiliza el IDE (Integrated Development Environment), que es el entorno de desarrollo o software que interactúa con la placa programable, el cual está basado en la programación de propósito general "C", por lo que se hace sencilla la manipulación de entradas y salidas de la placa (Hernández Mendoza, Serrano Rubio, \& De los Reyes Quiroz, 2019).

Los proyectos electrónicos, cuando se trata de distancias cortas, actualmente usan comunicación inalámbrica Bluetooth, que es una especificación industrial para redes inalámbricas de área personal creado por Bluetooth Special Interest Group y posibilita la transmisión de voz y datos entre diferentes dispositivos mediante un enlace por radiofrecuencia en la banda ISM (Industrial, Scientific and Medical) de los $2.4 \mathrm{GHz}$. En el presente, para la comunicación con un celular se usa el módulo Bluetooth HC-06 que se conecta mediante comunicación serial con la placa controladora (Silva Cruz et al., 2018).

La programación de aplicaciones móviles de manera muy sencilla se realiza utilizando MIT App Inventor, que es una plataforma para crear aplicaciones móviles en un navegador web (Annherys et al., 2015). Pero cuando se trata de hacer aplicaciones móviles mucho más robustas se utiliza el lenguaje Java y el entorno de desarrollo integrado Android Studio, entorno oficial para el desarrollo de aplicaciones para Android. Además del potente editor de códigos y las herramientas para desarrolladores, ofrece aún más funciones que aumentan su productividad durante la compilación de aplicaciones (Villarrubia et al., 2018).

SQLite es un sistema de gestión de base de datos optimizada, más no ser un cliente-servidor, siendo una biblioteca que permite ser integrada en el programa. Por su funcionamiento, es más usada en los móviles para las aplicaciones que requieran un almacén de datos al no tener conectividad al Internet en su funcionamiento (Arboleda \& Loyaga, 2018).

El científico de Londres Faraday (1791-1867), en 1831 descubrió el fenómeno de inducción magnética, a una bobina puede inducirse corriente eléctrica cuando los flujos magnéticos son variantes cerca de ella, situación que permite acercar una bobina a un motor eléctrico, el cual genera inducción magnética a su alrededor cuando esta alimentado, para verificar en qué momento inicia el funcionamiento del motor y en qué momento se detiene. Si esta corriente inducida se la conecta a un pin analógico del microcontrolador Arduino, podemos detectar el funcionamiento del motor por más pequeña que sea dicha corriente. El detector Bobina Rogowski puede detectar magnetismo a su alrededor dependiendo si es variante generar corriente en sus terminales, cosa que permitiría detectar el flujo magnético que genera un motor en movimiento. Si la bobina Rogowski forma un circuito cerrado en torno al flujo a ser medida, inducirá una tensión en los terminales de ésta, permitiendo que se conecten al microcontrolador y procesar dichos datos (Campos Montiel et al., 2009).

A su vez, el sensor LM35 es un sensor de temperatura de precisión en circuito integrado de tres terminales que posee un rango de temperatura de -55 a 155 grados centígrados. Cuando este es conectado, el pin de salida envía al microcontrolador una señal analógica en milivotios. El valor que se obtiene se almacena en una variable binaria que puede ser de 8 o 10 bits, según la 
configuración del controlador. Posteriormente, este valor se convierte a grados Celsius mediante la siguiente fórmula:

$$
C=(5 * \operatorname{analog} \operatorname{Read}(\operatorname{PinLm35}) * 100) / 1023
$$

Donde el 5 representa el voltaje máximo que envía el sensor, el 100 representa la conversión de $10 \mathrm{mV} /\left({ }^{\circ} \mathrm{C}\right)$ a $1 \mathrm{mV} /\left({ }^{\circ} \mathrm{C}\right)$. El 1023 representa el valor máximo con 10 bits en decimal. El LM35 fue colocado dentro o cerca del estator de cada motor, dependiendo del tipo de motor, para detectar la temperatura de funcionamiento del mismo (Zavala, 2015).

En varias empresas visitadas se pudo apreciar dificultades en la toma de decisiones con respecto a motores eléctricos de máquinas de producción, por ejemplo, no se tiene periodos determinados de mantenimiento, se tienen fallos de motores inesperados que detienen la producción y las empresas que poseen un sistema de captación tuvieron una inversión exagerada.

Para solucionar esta problemática, se propone el presente trabajo investigativo cuyo objetivo general es proponer un sistema para la captación de datos del funcionamiento de motores para la toma de decisiones en el departamento de producción en empresas del centro del país.

\section{Metodología}

El enfoque de la investigación fue mixto, ya que en parte del estudio se utilizó conteo numérico, tanto en la parte de la encuesta, como en el análisis de captación de datos y parte del estudio de métodos de recolección de datos es de tipo descriptivo para referir categorías conceptuales.

De acuerdo con el objetivo establecido se inició identificando el impacto que tuvo un sistema de captación de datos en las empresas de producción. Se detalló la realidad de situaciones y eventos en la captación de datos, por lo que, según el alcance, la investigación también fue descriptiva.

En la zona centro del país (Ecuador), una de las principales actividades económicas empresariales es la producción de prendas de vestir, calzado y carrocerías.

De acuerdo a lo anterior, las empresas objetivo fueron Plasticaucho, Calzado Jhon's, Vecachi, que producen calzado, IMPA, que produce carrocerías, y Curtiduría Promepell S.A., que produce cuero. Al ser preguntas técnicas, se realizó una entrevista a los 5 jefes técnicos de empresas de producción en el centro del país.

Los métodos científicos utilizados fueron la observación para verificar los problemas en las empresas de producción y el analítico sintético para realizar inferencias de datos estadísticos mediante la aplicación de la técnica de la entrevista. En la Tabla 1 se muestra el resumen de la misma. 
Tabla 1.

Resumen de la entrevista

\begin{tabular}{|l|l|l|l|}
\hline Número & Pregunta & Si & No \\
\hline 1 & $\begin{array}{l}\text { ¿Conoce usted las características técnicas del funcionamiento de } \\
\text { motores en su industria? }\end{array}$ & 5 & 0 \\
\hline 2 & $\begin{array}{l}\text { ¿Realiza usted pruebas periódicas del funcionamiento de los } \\
\text { motores en su industria? }\end{array}$ & 2 & 3 \\
\hline 3 & $\begin{array}{l}\text { ¿En su empresa disponen de algún sistema de captación de datos } \\
\text { de motores eléctricos para verificar su funcionamiento? }\end{array}$ & 1 & 4 \\
\hline 4 & $\begin{array}{l}\text { ¿El sistema aportó a la toma de decisiones sobre motores eléctricos } \\
\text { en su industria? }\end{array}$ & 1 & 4 \\
\hline 5 & $\begin{array}{l}\text { ¿Usted cree conveniente invertir en un nuevo sistema para captar } \\
\text { datos técnicos de motores en su industria? }\end{array}$ & 5 & 0 \\
\hline
\end{tabular}

Fuente: elaboración propia

En la pregunta número 1 se muestra el conocimiento de los jefes sobre las características técnicas que pueden afectar al funcionamiento de un motor.

La pregunta 2 evalúa si se realiza pruebas de funcionamiento de motores en las empresas de producción.

La pregunta 3 determina si en las empresas de producción tienen algún sistema de captación de datos para realizar los mantenimientos oportunamente a los motores eléctricos. La 4 es exclusiva para las personas que respondieron sí en la pregunta anterior, ya que evalúa si el sistema que poseen aporto en algo a la toma de decisiones.

Y la 5 valora si las empresas están dispuestas a invertir en un nuevo sistema que facilite tomar decisiones sobre el funcionamiento de motores eléctricos en sus industrias. La encuesta una vez realizada arrojó los siguientes resultados resumidos en la Figura 1.

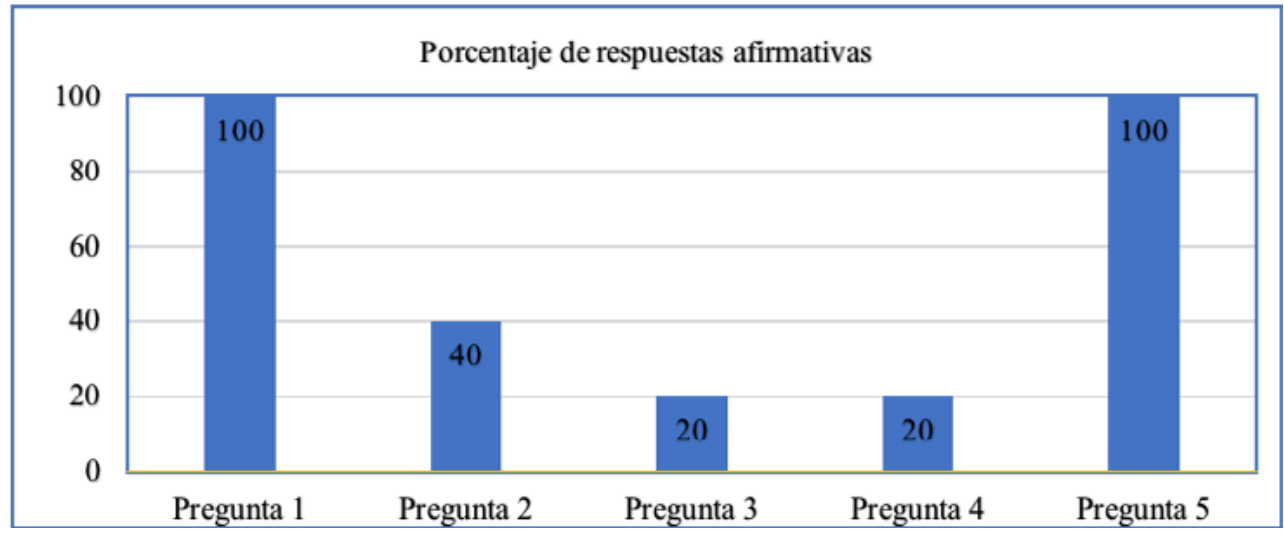

Figura 1. Resultados resumen entrevista.

Fuente: elaboración propia 
Analizando los resultados de la encuesta y entrevistas, se puede considerar que en las empresas que cuentan con al menos 3 motores en su planta de producción, el jefe técnico conoce los fallos inesperados que pueden detectarse en un motor censando sus variables, y que esto puede adelantar mantenimientos correctivos en los mismos, y que ellos consideran que, sería una buena inversión instalar un sistema que no dependa de las grandes empresas con costos elevados, que permita realizar el proceso de verificación en tiempo real, hacer comparaciones con datos anteriores y tener datos concretos sobre el funcionamiento de los mismos.

\section{Resultados}

En base a los resultados anteriores, se tiene la siguiente propuesta de solución. El sistema SADAM ayudará a los jefes técnicos a tomar decisiones en base a estadística con monitoreo, involucrando tres etapas plenamente marcadas, la primera que corresponde a la toma de datos desde los motores eléctricos en la industria utilizando bobinas y sensores de temperatura, la segunda que recibe los datos en un microcontrolador Arduino por conversor análogo - digital y su fácil programación para la captación y envío de datos mediante comunicación Bluetooth, comunicación que se utiliza, ya que los datos a enviar no son muy grandes y no se necesita mucha velocidad, ya que se hace muestreo cada cierto tiempo. Los datos son recibidos en una aplicación móvil en un dispositivo inteligente, la cual los muestra y almacena, además, también los procesa para tomar decisiones sobre el funcionamiento de los motores en la industria. La Figura 2 muestra la interacción de cada una de las etapas.

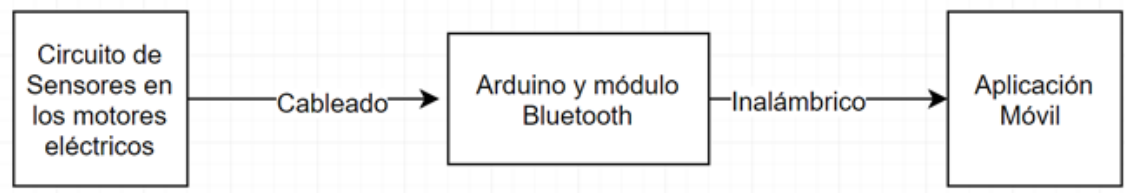

Figura 2. Diagrama de bloques del sistema SADAM

Fuente: elaboración propia

Para la toma de datos de los motores eléctricos se puede trabajar con varios sensores, para el prototipo planteado se consideran dos, el tiempo de funcionamiento (Bobina detectora) y otro de temperatura de trabajo (LM35).

Los diferentes sensores utilizados están interconectados en una misma placa, la cual con cable no mayor a 5 metros llega al Arduino que posteriormente mediante comunicación Bluetooth llega a la aplicación creada para Android.

El la Figura 3 se observa el circuito realizado como prototipo, en el cual se tiene la conexión de seis bobinas y seis sensores de temperatura, con sus respectivas alimentaciones y conexiones. 


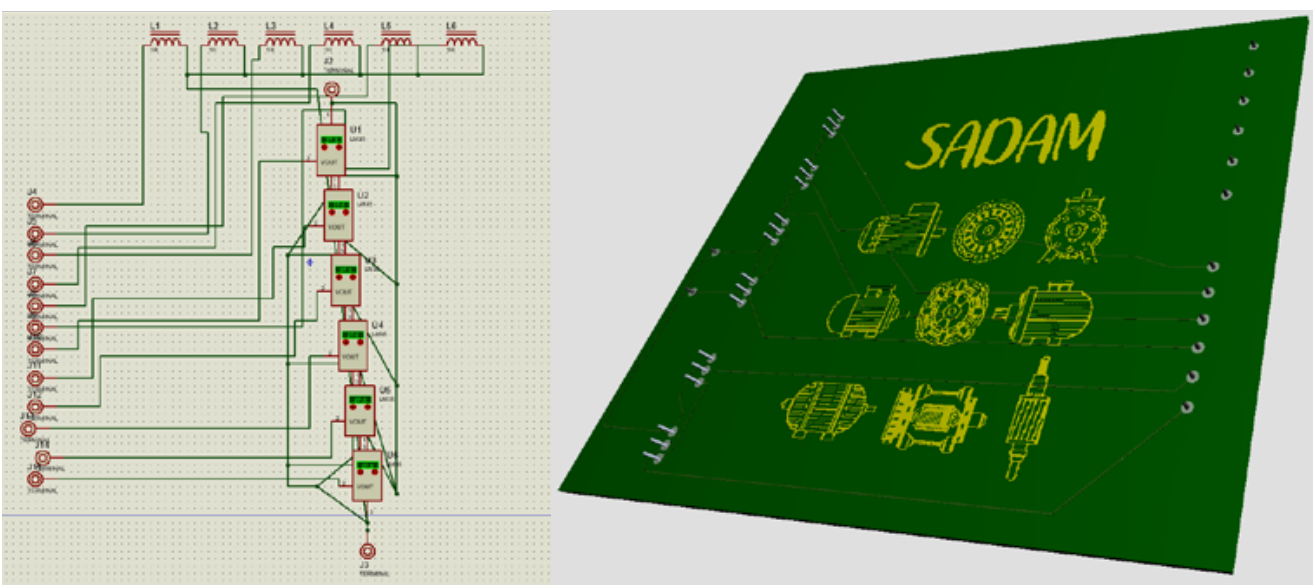

Figura 3. Circuito Simulado y placa de los sensores

Fuente: elaboración propia

La unidad de control utilizada en el prototipo es el Arduino Mega, el cual permite en sus pines analógicos recibir los datos desde los sensores, los procesa y los envía a la aplicación móvil a través del módulo Bluetooth por sus pines de comunicación Serial. Para la implementación del prototipo se utilizó el módulo HC-06 junto con una placa Arduino Mega, por la cantidad de entradas analógicas que se necesitan, a su vez, se implementó el entorno de desarrollo de Arduino para configurar el módulo Bluetooth como servidor y también los parámetros necesarios para su identificación y funcionamiento: velocidad de ciclo de reloj, nombre del dispositivo Bluetooth y contraseña de acceso, las conexiones realizadas del circuito son las que se muestra en la Figura 4.

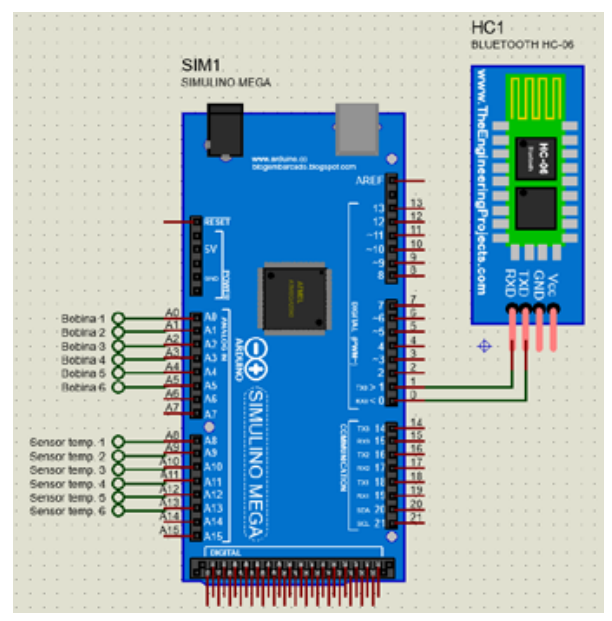

Figura 4. Conexiones del Arduino y módulo Bluetooth

Fuente: Elaboración propia

La aplicación móvil desarrollada se llama "SADAM" por las siglas de Sistema de Adquisición de Datos de Motores. Esta recibe los datos tomados por los sensores desde el Arduino a través de la comunicación Bluetooth, en la Figura 5 se muestra la pantalla principal de la aplicación desarrollada en el software Android Studio. 


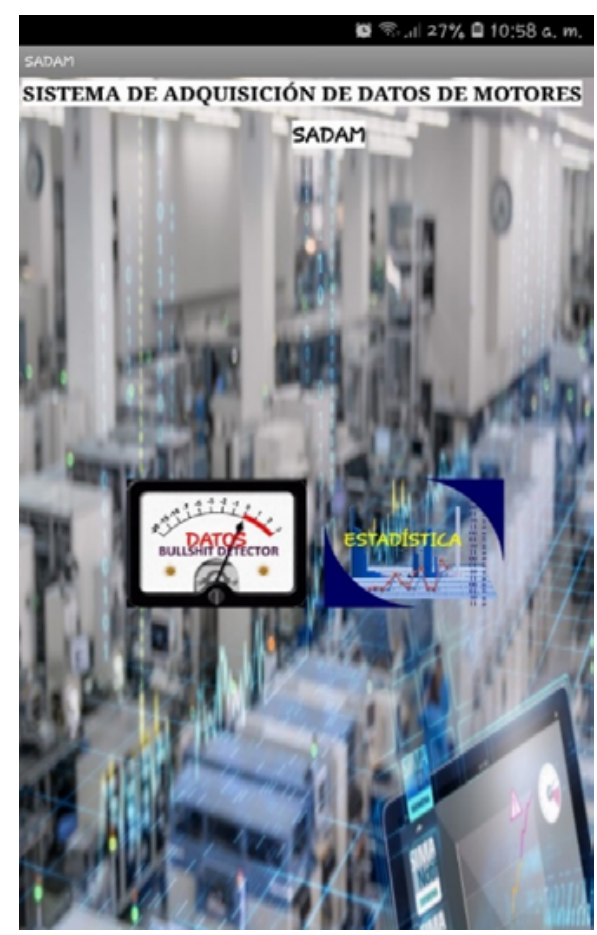

Figura 5. Pantalla principal del SADAM

Fuente: elaboración propia

En la pantalla principal se tiene dos botones llamados datos y estadística. El primero abre una ventana donde se muestran datos al instante con una frecuencia para la toma de sesenta segundos, los cuales se los toma como referencia, ya que el cambio de las variables censadas no es tan repentino, y con un periodo de muestreo de 1 minuto es suficiente para el correcto funcionamiento el sistema. En la Figura 6 se muestra la pantalla cuando se activa el botón datos, donde se evidencian los datos sin proceso estadístico, y el botón estadística, donde se muestran los resultados del funcionamiento de los motores, para poder tomar decisiones sobre los mismos.

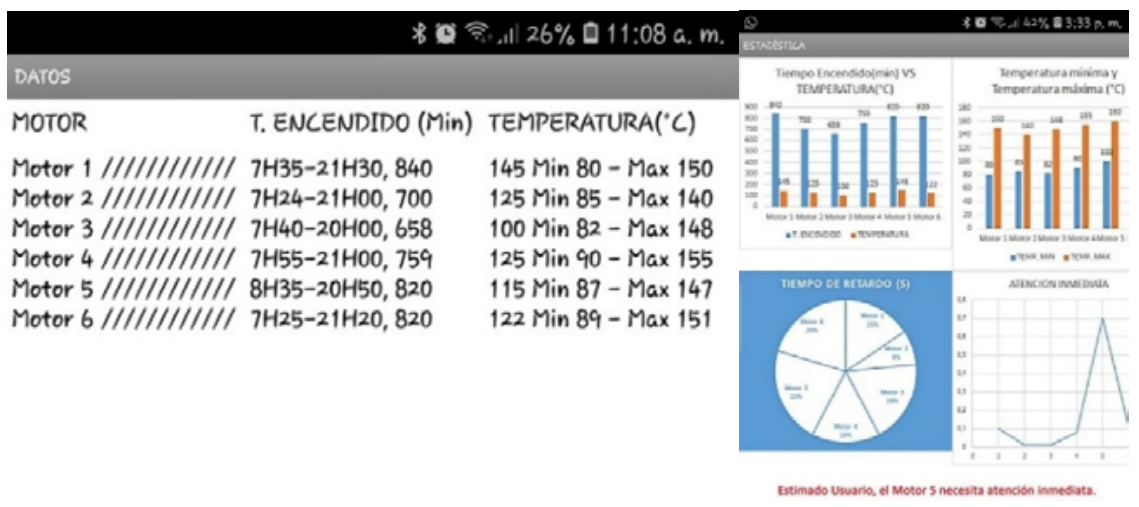

Figura 6. Pantalla de datos y estadística del sistema SADAM

Fuente: elaboración propia 
En la Figura 6 se pueden ver los datos de tiempo de encendido, tiempo de retardo al arranque y la estadística de los 6 motores evaluados con el prototipo.

En la parte de datos como se muestran, no permiten tener un criterio muy considerable sobre algún valor erróneo o alarmante de los motores, ya que son datos crudos, los cuales no representan información útil para el operador. Pero en la parte de estadística tenemos ya un análisis comparativo y estadístico de los datos iniciales, la primera gráfica de barras de doble entrada de tiempo de encendido versus temperatura, la cual nos puede dar a conocer valores precisos sobre el tema que un motor se calienta mientras más se utiliza, además, depende de la carga que se le coloque. Un segundo gráfico de barras de doble entrada de temperatura mínima y temperatura máxima, que está evaluado por semanas, que comparando con valores antiguos o preestablecidos según el fabricante pueden darnos ya a conocer sobre un problema en un motor que exceda la temperatura máxima o disminuya demasiado la temperatura mínima.

Además, tenemos un gráfico tipo pastel, que evalúa el tiempo de retardo al arranque desde el momento que se enciende el motor, qué tiempo tarda en arrancar, claro, considerando la carga a la que este expuesto, y el tipo de arranque que modificará los datos a los cuales serán comparados, esto nos puede igualmente mostrar datos de algún inconveniente en el funcionamiento regular de un motor.

Y, por último, tenemos un gráfico lineal, que evidencia un resumen total, ya que evalúa y compara todos los datos tomados y los muestra en una sola gráfica, la cual manifiesta qué motor debería ser atendido de inmediato, prácticamente sigue funcionando, pero ya presenta varios valores anormales en su funcionamiento, esta grafica también tiene una alerta que se muestra en la parte inferior de la misma. Las representaciones pueden ser modificadas según los requerimientos del usuario y las variables normales de funcionamiento provistas por el fabricante o valores modificados por experiencia del jefe técnico, para realizar mejor las comparaciones y presentar los datos de mejor manera.

En las pruebas realizadas se utilizaron distintos tipos de motores eléctricos para poder usar todas las características de nuestro sistema, en el Motor 5 se colocó intencionalmente uno que tiene inconvenientes en cuanto al funcionamiento. Por tal razón en la Figura 6, en la parte correspondiente, nos muestra que el motor 5 necesita atención inmediata. Todas las gráficas y alarmas se pueden modificar de acuerdo a los requerimientos.

El resultado del presente artículo es el correcto funcionamiento del prototipo de un sistema de adquisición de datos del funcionamiento de motores eléctricos en la industria (SADAM), el cual será una gran competencia bajando costos y siendo más específicos a los requerimientos deseados, a los grandes sistemas de adquisición de datos (SCADA) ofertados por grandes empresas a nivel mundial como Labview, Siemens, Bosch, etc.

Al sistema implementado se le puede añadir más sensores para tomar datos específicos, como se muestra en la Figura 4, podemos utilizar los pines digitales y de comunicación I2C del Arduino, pero el prototipo creado toma tiempo y temperatura de funcionamiento, y los compara con criterios de funcionamiento preestablecidos en cuanto a marcas, antigüedad, carga, etc. Para verificar su 
funcionalidad, el sistema permite verificar datos al instante con una frecuencia de un minuto, y además brinda estadística del funcionamiento por semanas.

El uso del sistema en el proceso de captación de datos de motores en empresas de producción supone una gran ventaja frente a las demás empresas. Sobre todo, por la propuesta de un sistema que tome datos específicos de los motores sin afectar a la fabricación continua, ya que no se necesita parar la producción para su instalación.

Empresas como TRIMAX, IGNITION, SIEMENS o MYSCADA dan solución a través de una conexión WIFI con la central SCADA. Pero para esto se necesita primero la instalación completa del sistema SCADA, cuestión que para empresas de mediana producción no es accesible, es ahí donde entra la propuesta del sistema SADAM, ya que dicho sistema puede o no tener algún sistema previo instalado y funcionar perfectamente, ya que se lo instala sin modificar los servicios previos en la planta de producción (TRIMAX, 2013).

Comparando resultados con los mostrados en el estudio de Fernández de Córdova Álvarez y Vanegas (2018), se puede apreciar una similitud considerando que en ese caso se toma datos de humedad del suelo y los envía a un dispositivo móvil para poder tomar decisiones acerca del de la humedad del suelo.

Así también en el artículo de Ruiz-Ayala, Vides-Herrera, \& Pardo-García (2018) se monitorea variables meteorológicas a través de un sistema de adquisición de datos, lo que permite realizar estadística y pronosticar cambios climáticos como en nuestro sistema al pronosticar fallos de motores. Además, el trabajo de Pontes, Gavilán, Obrero y Flores (2006) realizó captación de datos para realizar una plataforma educativa, y en el estudio de Amador-Álvarez (2017) se toma datos para la toma de decisiones en un sistema hidropónico de lechuga en un invernadero.

Como se puede evidenciar, existe una relación directa en la captación de datos y toma de decisiones, lo que permitiría expandir el sistema a diferentes empresas de producción y no solo evaluar motores eléctricos, sino las distintas variables en sensores y actuadores en la etapa de producción de las empresas.

Además, podría trasladarse el sistema SADAM a sistemas domóticos que brinden seguridad, confort, ahorro energético y comunicaciones simplemente modificando la placa de sensores y utilizando salidas digitales del microcontrolador para utilizar actuadores, dependiendo del tipo de variables que se deseen controlar.

\section{Conclusiones}

En el presente trabajo se generó un sistema integral de software y hardware para la captación de datos el cual permite al gerente o jefe técnico tomar decisiones sobre el cambio de motores eléctricos o mantenimiento de los mismos.

Los sistemas SCADA móviles actuales permiten liberar a los operadores de sus estaciones de trabajo ya que pueden acceder a SCADA desde cualquier dispositivo móvil, ya sea en el sitio o incluso desde una ubicación remota para acelerar la notificación de alarmas, reducir el tiempo 
de inactividad y mantener su sistema funcionando sin problemas. Pero estos necesitan tener el sistema SCADA completo para usar la herramienta móvil.

EI sistema SADAM, tanto en hardware y software, utiliza comunicación alámbrica y Bluetooth conjuntamente, se puede modificar según los requerimientos solicitados, además, no es necesario tener SCADA instalado, ya que es un sistema independiente.

Este es un método que alerta problemas, más no los corrige; es decir, es un sistema de adquisición de datos, más no de control, pero claro, además de adquirir datos, realiza una estadística de funcionamiento para presentarlo como posibles pronósticos de fallos en los motores eléctricos y en sí correcciones, cambios o mantenimiento de los mismos que se deban realizar con la brevedad del caso dependiendo de los datos mostrados.

Para proyectos posteriores se puede pensar en diseñar e implementar un dispositivo similar pero que funcione mediante la tecnología Wifi, también podemos pensar en modificaciones que integren inteligencia artificial al diseño actual o un software para programar efectos mediante una interfaz gráfica sin necesidad de utilizar estadística.

EI sistema SADAM puede ser utilizado en la industria farmacéutica, supermercados, alimenticia, textiles, petrolífera, etc., simplemente modificando sus sensores, para medir distintas variables físicas en los distintos equipos o elementos en las plantas de producción. 


\section{Referencias}

Amador-Álvarez, A. (2017). Diseño de un sistema electrónico inalámbrico de adquisición de datos para la toma de decisiones en un sistema hidropónico de lechuga en un invernadero del TEC sede San Carlos. https:// hdl.handle.net/2238/9857

Annherys, P., \& Jaiham, H \& García, J. (2015). Toma de decisiones: reto para crear ventajas competitivas en las distribuidoras de alimentos gourmet. Desarrollo Gerencial. 7(2), 100-118. https://doi.org/10.17081/ dege.7.2.1183

Arboleda, J., \& Loyaga, W. (2018). Análisis, diseño y construcción de un sistema web para realizar cotizaciones de acero en la empresa Novacero SA y una aplicación android dirigida para los asesores de ventas en la ciudad de Quito-Ecuador. Tesis. https://dspace.ups.edu.ec/handle/123456789/16033

Campos Montiel, S., Carranza López, R \& González Parada, A. (2009). Bobina Rogowski como sensor de corriente para monitoreo de transformadores de distribución. Encuentro Nacional de Metrología Eléctrica 2009. https://www.cenam.mx/dme/pdf/EXT T1-Jue-2.pdf

Gálvez, C. (2018). Solución móvil para el monitoreo de plantas mineras en tiempo real. http://repositorio.usil. edu.pe/handle/USIL/3095

Fernández de Córdova Álvarez, C. P., \& Vanegas Verdugo, D. H. (2018). Diseño e implementación de un sistema SCADA para el control de riego mediante un dispositivo móvil (Bachelor's thesis, Universidad del Azuay). http://dspace.uazuay.edu.ec/bitstream/datos/7911/2/13651.pdf

Hernández Mendoza, C. M., Serrano Rubio, J. P., \& De los Reyes Quiroz, F. (2019). Conectividad en redes inalámbricas de área personal utilizando Arduino y componentes electrónicos / Connectivity in Personal Área Wireless Networks Using Arduino and Electronic Components. RECI Revista Iberoamericana de Las Ciencias Computacionales e Informática, 8(15), 45. https://doi.org/10.23913/reci.v8i15.93

Ibarra Cisneros, M., González Torres, L \& Demuner Flores, M. (2017). Competitividad empresarial de las pequeñas y medianas empresas manufactureras de Baja California. Estudios Fronterizos, 18(35), 107-130. https://doi.org/10.21670/ref.2017.35.a06

Marcano Aular, J \& Talavera Pereira, R. (2007). Minería de Datos como soporte a la toma de decisiones empresariales. Opción, 23(52), 104-118. http://ve.scielo.org/scielo.php?script=sci arttext\&pi$\underline{d}=\mathbf{S} 1012-15872007000100008$

Mariño, P., Poza, F., Ubeira, M., \& Machado, F. (2004). Sistema de Adquisición y Almacenamiento de Datos para Monitorización del Estado de Transformadores de Potencia. Información Tecnológica, 15(2), 95-100. https://doi.org/10.4067/S0718-07642004000200017 
Martell, L. B., Paneque, R. C. M., \& Cuesta, B. V. (2016). Sistemas SCADA para la automatización de los procesos productivos del CIGB. Ingeniería Electrónica, Automática y Comunicaciones, 1, 20-37. http://scielo. sld.cu/scielo.php?script=sci arttext\&pid=S1815-59282016000100003

Millahual, C. P. (2017). Arduino - De Cero a Experto: Proyectos Prácticos - Electrónica, hardware y programación. RedUsers. https://books.google.com.ec/books?id=8PiEDwAAQBAJ

Pérez-López, E. (2015). Los sistemas SCADA en la automatización industrial. Revista Tecnología En Marcha, 28(4), 3-14. https://doi.org/10.18845/tm.v28i4.2438

Pontes, A., Gavilán, J., Obrero, M \& Flores, A. (2006). Diseño y aplicación educativa de un programa de simulación para el aprendizaje de técnicas experimentales con sistemas de adquisición de datos. Revista Eureka, 3(2) https://www.redalyc.org/pdf/920/92030207.pdf

Ruiz-Ayala, D. C., Vides-Herrera, C. A., \& Pardo-García, A. (2018). Monitoreo de variables meteorológicas a través de un sistema inalámbrico de adquisición de datos. Revista De Investigación, Desarrollo e Innovación, 8(2), 333. https://doi.org/10.19053/20278306.v8.n2.2018.7971

Salazar, J. Á., \& Arango, J. G. M. (2017). TIA Portal. Aplicaciones de PLC. Textos Académicos. https://books. google.com.ec/books?id=aOUzDwAAQBAJ

Silva Cruz, F., Caballero Julián, F., Silva, M., Huerta, C \& Velásquez Cruz, I. (2018). Caracterización de la función de transferencia del llenado de un tanque, aplicando un sistema IOT, conformado por un sensor ultrasónico, la plataforma arduino y una aplicación móvil (characterization of the transfer function of the filling of a tank, applying a system IOT, conformed by an ultrasonic sensor, the arduino platform and a mobile application). Pistas educativas. 40 (130). http://itcelaya.edu.mx/ojs/index.php/pistas/article/view/1648

Thompson, S. (2019). Arduino: 8 proyectos divertidos para aprender sin darte cuenta. Steadman Thompson. https://books.google.com.ec/books?id=UJqNDwAAQBAJ

TRIMAX. (2013). SCADA/HMI. http://www.trimaxsystems.com/solutions/mobile-scada.html

Villarrubia, Z. A., Gracia, C. F., Núñez, R. P., Martínez, C. H., Aparicio, R. P., Hierro, A. S., \& García, D. F. (2018). Radiación en Medicina: Aplicación para dispositivos Android. Revista de Física Médica, 19(1), 55-60.

Zavala, J \& Márquez, J. (2015). Sistema integral de software y hardware para el aprendizaje del funcionamiento y manejo de los sensores. Pistas Educativas, 36 (112), 895-917. http://itcelaya.edu.mx/ojs/index.php/ pistas/article/view/414 
Copyright (c) 2021 Angel Arturo Flores Lescano

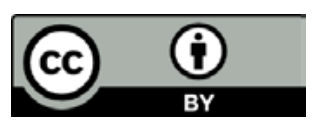

Este texto está protegido bajo una licencia internacional Creative Commons 4.0.

Usted es libre para Compartir-copiar y redistribuir el material en cualquier medio o formato - y Adaptar el documento - remezclar, transformar y crear a partir del material-para cualquier propósito, incluso para fines comerciales, siempre que cumpla las condiciones de Atribución. Usted debe dar crédito a la obra original de manera adecuada, proporcionar un enlace a la licencia, e indicar si se han realizado cambios. Puede hacerlo en cualquier forma razonable, pero no de forma tal que sugiera que tiene el apoyo del licenciante o lo recibe por el uso que hace de la obra.

Resumen de licencia - Texto completo de la licencia 